\title{
ANALYSIS OF POSSIBLE SCATTERING PROCESSES FOR BLOCH POSITRONIUM IN IONIC CRYSTALS AT ELEVATED TEMPERATURES
}

\author{
I.V. BONDAREV
}

The Institute of Nuclear Problems, The Belarusian State University Bobruiskaya Str. 11, 220050 Minsk, Belarus

\begin{abstract}
The role of nonpolar optic phonon scattering and of umklapp acoustic phonon scattering is analyzed for a delocalized positronium atom in ionic crystals at elevated temperatures. At temperatures above which they become essential both of these scattering mechanisms contribute to the imaginary self-energy of positronium renormalizing its acoustic deformation potential constant. It was such a renormalization that was recently observed experimentally for delocalized positronium in $\mathrm{MgF}_{2}$ crystal. We put forward the theoretical explanation of these experiments based upon the model of nonpolar optic phonon scattering of positronium. An effect analogous to that observed in $\mathrm{MgF}_{2}$ is predicted for crystalline quartz above the temperature of $(\alpha-\beta)$-phase transition.
\end{abstract}

PACS numbers: 78.70.Bj, 71.60.+z, 71.38.+i, 36.10.Dr

\section{Introduction}

Positronium (Ps), the bound state of an electron and a positron, has been found to be formed in a delocalized Bloch-type state in ionic crystals with a low enough concentration of defects at sufficiently low temperatures (typically below a few tens K) [1]. The formation of Bloch-type Ps in these crystals is confirmed by observing very narrow peaks (the central peak and satellite peaks appearing at the momentum corresponding to the reciprocal lattice vectors of the sample crystal) in the momentum distribution of the photons resulting from the $2 \gamma$-decay of Ps upon irradiating the crystals by low-energy positrons. As temperature increases, it is observed that the central Ps peak becomes drastically wider and the satellite peaks disappear, indicating the localization of Ps [1]. Such an effect of a thermally activated self-localization of Ps was observed in many ionic crystals and was analyzed theoretically in Refs. $[2,3]$. The only exceptions presently known are the 
crystals of $\mathrm{MgF}_{2}$ and $\alpha-\mathrm{SiO}_{2}$ ( $\alpha$-phase of crystalline quartz). As is shown in recent experiments $[4,5]$, the Ps atom in these crystals remains delocalized up to temperatures $\sim 700 \mathrm{~K}$ (this experimental fact was first explained theoretically in Ref. [3]). The central and satellite Ps peaks in $\mathrm{MgF}_{2}$ are observed to be drastically broadened at temperatures higher than $200 \mathrm{~K}$. This drastic broadening was failed to be explained in terms of Ps scattering on longitudinal acoustic phonons. An effect appeared as if there were an additional scattering mechanism activated at temperatures higher than $200 \mathrm{~K}$ which renormalized the acoustic deformation-potential constant of Ps so that it increased by a factor of approximately two in the narrow temperature range from 200 to $355 \mathrm{~K}$. A similar effect was not observed in $\alpha-\mathrm{SiO}_{2}$ where the temperature broadening of the central and satellite peaks of the Ps momentum distribution was satisfactorily explained by means of Ps-acoustic-phonon scattering throughout the entire temperature range of $\sim 80-700 \mathrm{~K}$.

In terms of the Green functions formalism [6], the Ps momentum distribution at finite temperatures is expressed as $[4,5]$

$$
N(p) \sim \int_{0}^{\infty} \mathrm{d} \omega \exp \left(-\omega / k_{\mathrm{B}} T\right) \frac{\Gamma(p, \omega)}{\left(\omega-p^{2} / 2 M^{*}\right)^{2}+\Gamma^{2}(p, \omega)},
$$

where the exponential factor stands for the Boltzmann statistics because there is at most only one Ps atom at a time under usual experimental conditions. The non-exponential factor represents the so-called spectral density function in its explicit form with $\Gamma(p, \omega)$ being the imaginary self-energy of Ps [6]. In the weak phonon coupling regime (delocalized Ps) the latter one is usually written to the lowest (second) order approximation in the Ps interaction with a phonon field:

$\Gamma(p, \omega)=\pi \sum_{\mathbb{Q}}\left|V_{\mathbb{Q}}\right|^{2}\left[\left(n_{\mathbb{Q}}+1\right) \delta\left(\omega-E_{\boldsymbol{k}+\mathbb{Q}}-\hbar \omega_{\mathbb{Q}}\right)+n_{\mathbb{T}} \delta\left(\omega-E_{\boldsymbol{k}+\mathbb{T}}+\hbar \omega_{\mathbb{U}}\right)\right.$,

where $V_{\boldsymbol{q}}$ is the interaction matrix element, $E_{\boldsymbol{k}}=\hbar^{2} \boldsymbol{k}^{2} / 2 M^{*}$ the energy of Ps with the band mass $M^{*}$ and quasimomentum $k=p / \hbar, n_{\mathbb{q}}=\left[\exp \left(\hbar \omega_{\mathbb{Q}} / k_{\mathrm{B}} T\right)-1\right]^{-1}-$ the equilibrium phonon distribution function, $\omega_{\mathbb{q}}$ - the frequency of the phonon with the wave vector $q$.

The interaction energy of a particle with lattice vibrations in its simplest form depends linearly on strain - acoustic strain in the case of acoustic phonon modes and optic strain in the case of optic phonon modes (see, for instance, Ref. [7]). These strains influence the particle in its band in two distinct ways. In the first way, short-range disturbances of the periodic potential cause practically instantaneous changes in energy, and these are the ones quantified by deformation potentials and referred to as deformation-potential scattering (acoustic and nonpolar optic, respectively). In the second way, the distortion of the lattice may destroy local electric neutrality, and produce electric polarization and associated macroscopic comparatively long-range electric fields to which the particle responds. Disturbance of the particle's motion by this effect is referred to as piezoelectric scattering, if associated with acoustic modes, and polar optic scattering, if associated with optic modes. Contrary to deformation-potential scattering, the latter two are believed to be less important for the Ps atom in ionic crystals because of its electric neutrality [8]. 
Acoustic deformation-potential scattering is described by the Ps-acoustic-phonon interaction with the Hamiltonian given in the simplest form by [7,9]

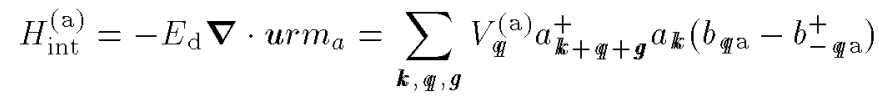

with $E_{\mathrm{d}}$ and $u_{\mathrm{a}}$ being the acoustic deformation-potential constant and acoustic lattice displacement, respectively. The right-hand side of the equation represents the secondly quantized form of the interaction, where $a_{k_{k}}^{+}\left(a_{\text {k }}\right)$ and $b_{\text {qa }}^{+}\left(b_{q \mathrm{q}}\right)$ are the creation (annihilation) operators for Ps and longitudinal acoustic phonons, respectively, $\boldsymbol{g}$ is such a reciprocal lattice vector that $\boldsymbol{k}$ and $\boldsymbol{k}^{\prime}=\boldsymbol{k}+\boldsymbol{q}+\boldsymbol{g}$ belong to the first Brillouin zone of a crystal. The interaction matrix element takes the form $[3]$

$$
\frac{V_{\mathbb{Q}}^{(\mathrm{a})}=-\mathrm{i} E_{\mathrm{d}} \sqrt{\frac{\hbar}{2 N M \omega_{\mathbb{q} \mathrm{a}}}} q}{\left[1+\left(q a_{\mathrm{B}} / 4\right)^{2}\right]^{2},}
$$

where $N$ is the number of unit cells in the crystal, $M$ - the total mass of the atoms in the unit cell, $a_{\mathrm{B}}$ - the Bohr radius of Ps, and $q$ - the absolute value of $q$.

The interaction $H_{\text {int }}^{(\mathrm{a})}$ consists of two parts. The term with $\boldsymbol{g}=0$ describes the normal scattering processes on long-wave longitudinal acoustic phonons of the frequency $\omega_{\mathbb{q} \text { a }}=u q$, where $u$ is the average velocity of longitudinal acoustic vibrations. Being substituted in Eq. (2), this term yields the Ps imaginary self-energy in the form

$$
\Gamma^{(\mathrm{a})}(p, \omega)=\frac{E_{\mathrm{d}}{ }^{2} M^{* 3 / 2} k_{\mathrm{B}} T}{\sqrt{2} \pi \hbar^{3} u^{2} \rho} \sqrt{\omega},
$$

where $\rho$ is the density of the crystal, $k_{\mathrm{B}}$ - the Boltzmann constant. Equation (1) with the allowance made for Eq. (5) satisfactorily explains the temperature dependence of the $\mathrm{Ps}$ momentum distribution in $\alpha-\mathrm{SiO}_{2}$ and fails in explaining it in $\mathrm{MgF}_{2}$ at temperatures above $200 \mathrm{~K}$. In the latter case, one has to assume the increase in the $E_{\mathrm{d}}$ constant with temperature in the narrow temperature range of $200-355 \mathrm{~K}$ so that it changes from $7.6 \mathrm{eV}$ below $200 \mathrm{~K}$ to $16 \mathrm{eV}$ above $355 \mathrm{~K}[4,5]$.

The term with $g \neq 0$ describes so-called umklapp scattering on short-wave acoustic phonons [10]. This term yields the correction to Eq. (5) which renormalizes the acoustic deformation-potential constant $E_{\mathrm{d}}$ so that at temperatures above $T_{1}=\hbar \omega_{1} / k_{\mathrm{B}} \lesssim T_{\mathrm{D}}\left(\omega_{1}=2 u / a \lesssim \omega_{\mathrm{D}}\right.$ is the frequency of the short-wave acoustic phonon with the wave vector $\boldsymbol{q} \sim \boldsymbol{g} / 2 ; T_{\mathrm{D}}, \omega_{\mathrm{D}}$, and $a$ are the Debye temperature, Debye frequency, and lattice constant of a crystal, respectively) the renormalized effective constant $\widetilde{E}_{\mathrm{d}}$ starts growing with temperature in a sigmoidal way from $E_{\mathrm{d}}$ to $\sqrt{E_{\mathrm{d}}^{2}+\left(u E_{\mathrm{d}}^{(1)} / \omega_{1}\right)^{2}}$, where $E_{\mathrm{d}}^{(1)} \simeq \nu E_{\mathrm{d}} g / 2$ is the analog of the deformation-potential constant for umklapp scattering, $\nu$ - the number of the nearest neighbors in the reciprocal lattice space (see Ref. [10] for details). Such temperature behavior of $\widetilde{E}_{\mathrm{d}}$ is consistent with what was experimentally measured in $\mathrm{MgF}_{2}$ [4]. For this reason the mechanism of umklapp Ps-acoustic-phonon scattering was used in Ref. [5] as a model for theoretical interpretation of $\mathrm{MgF}_{2}$ data. However, if the umklapp effect is the case, then there arises and remains open the 
question why this scattering mechanism was not observed in $\alpha-\mathrm{SiO}_{2}$. In addition, the absorption or emission of the short-wave acoustic phonon by Ps would, according to Eq. (2), yield its intermediate-state energy $\omega$ in Eq. (1) of the order of $\omega \sim E_{\boldsymbol{k}+g / 2} \pm \hbar \omega_{1} \sim \hbar^{2}(g / 2)^{2} / 2 M^{*} \pm \hbar \omega_{1} \sim \hbar \omega_{\mathrm{D}} / \gamma \pm \hbar \omega_{\mathrm{D}} \sim \hbar \omega_{\mathrm{D}} / \gamma$, where $\gamma=$ $\hbar \omega_{\mathrm{D}} /\left(\hbar^{2} q_{\mathrm{D}}^{2} / 2 M^{*}\right)$ is the ratio of the maximal phonon energy to the particle's band width $\left(q_{\mathrm{D}}=\omega_{\mathrm{D}} / u \sim g / 2\right.$ is the Debye wave vector $)$, called the non-adiabaticity parameter [11]; $\gamma \sim 0.01$ for typical ionic crystals. Then the exponential factor in Eq. (1) would be of the order of $\exp \left(-\omega_{\mathrm{D}} / \gamma k_{\mathrm{B}} T\right) \sim \exp \left(-100 T_{\mathrm{D}} / T\right) \sim 0$ up to $T \sim 10^{4} \mathrm{~K}$, thereby completely cutting off the umklapp contribution in the momentum distribution of Ps.

The present paper suggests a novel approach to the theoretical explanation of the $\mathrm{MgF}_{2}$ and $\alpha-\mathrm{SiO}_{2}$ experimental data reported in Refs. [4, 5]. An advantage of this approach is that it explains both drastic temperature broadening of the Ps momentum distribution in $\mathrm{MgF}_{2}$ above $200 \mathrm{~K}$ and evidence for the absence of such broadening in $\alpha-\mathrm{SiO}_{2}$. The explanation is based upon the mechanism of optic deformation-potential scattering of Ps.

\section{Optic deformation-potential scattering and its role for Ps in ionic crystals}

Nonpolar optic deformation-potential-type phonon scattering is known to be of importance in crystals with two and more atoms per unit cell when a corresponding optic deformation-potential constant is non-zero because of local symmetry restrictions $[7,12,13]$. Indeed, although the number of acoustic phonons is much larger than that of optic phonons, the acoustic scattering matrix element squared is estimated to be of the order of that for nonpolar optic scattering multiplied by the small factor $q a \ll 1[12,13]$. To take the nonpolar optic scattering mechanism into consideration in the Ps problem we start with the interaction Hamiltonian of the form $[7]$

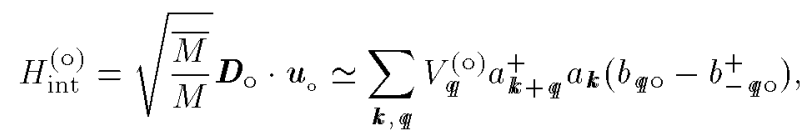

where $\boldsymbol{D}_{\circ}$ is the vectorial optic deformation-potential constant, $\boldsymbol{u}_{\mathrm{O}}$ - the optic lattice displacement, $\bar{M}$ - the reduced mass of a pair of atoms in the unit cell. The right-hand side of the equation represents the secondly quantized form of $H_{\text {int }}^{(o)}$ written within an isotropic approximation with umklapp processes neglected (compare with Eq. (3)). The operators $b_{\mathscr{q} 0}^{+}\left(b_{q 0}\right)$ create (annihilate) long-wave optic phonons of the constant frequency $\omega_{\circ}$,

$$
V_{\mathbb{q}}^{(\circ)}=-\mathrm{i} D_{\circ} \sqrt{\frac{\hbar}{2 M N \omega_{\circ}}}
$$

with $D_{\circ}$ being the absolute value of $\boldsymbol{D}_{\circ}$

Substituting the interaction matrix element Eq. (7) in Eq. (2), upon straightforward calculations one gets the nonpolar optic contribution to the Ps imaginary self-energy in the following form: 


$$
\begin{aligned}
& \Gamma^{(\circ)}(p, \omega)=\frac{D_{\mathrm{o}}^{2} M^{* 3 / 2} \sqrt{\omega}}{2 \sqrt{2} \pi \hbar^{2} \rho \omega_{\mathrm{o}}} \\
& \quad \times\left[\left(n\left(\omega_{\mathrm{o}}\right)+1\right) \theta\left(1-\frac{\hbar \omega_{\mathrm{o}}}{\omega}\right) \sqrt{1-\frac{\hbar \omega_{\mathrm{o}}}{\omega}}+n\left(\omega_{\mathrm{o}}\right) \sqrt{1+\frac{\hbar \omega_{\mathrm{o}}}{\omega}}\right]
\end{aligned}
$$

where $n\left(\omega_{0}\right)=\left[\exp \left(\hbar \omega_{\circ} / k_{\mathrm{B}} T\right)-1\right]^{-1}$ and $\theta(x)$ are the optic phonon distribution function and step function, respectively. Then the total imaginary self-energy accounting for both acoustic and nonpolar optic scattering of Ps can, in view of Eq. (5), be written as

$$
\Gamma(p, \omega)=\Gamma^{(\mathrm{a})}(p, \omega)+\Gamma^{(\mathrm{o})}(p, \omega)=\frac{\widetilde{E}_{\mathrm{d}}^{2}(\omega) M^{* 3 / 2} k_{\mathrm{B}} T}{\sqrt{2} \pi \hbar^{3} u^{2} \rho} \sqrt{\omega}
$$

with

$$
\begin{gathered}
\widetilde{E}_{\mathrm{d}}(\omega)= \\
\left\{E_{\mathrm{d}}^{2}+\frac{\hbar u^{2} D_{\mathrm{o}}^{2}}{2 k_{\mathrm{B}} T \omega_{\mathrm{o}}}\left[\left(n\left(\omega_{\mathrm{o}}\right)+1\right) \theta\left(1-\frac{\hbar \omega_{\mathrm{o}}}{\omega}\right) \sqrt{1-\frac{\hbar \omega_{\mathrm{o}}}{\omega}}+n\left(\omega_{\mathrm{o}}\right) \sqrt{1+\frac{\hbar \omega_{\mathrm{o}}}{\omega}}\right]\right\}^{1 / 2}
\end{gathered}
$$

representing the "effective" deformation-potential constant with nonpolar optic scattering taken into account. In view of the fact that only $\omega \sim k_{\mathrm{B}} T$ mainly contributes to the Ps momentum distribution (Eq. (1)), the $\omega$-dependence of $\widetilde{E}_{\mathrm{d}}(\omega)$ can be approximately changed by $T$-dependence so that Eq. (10) is rewritten in the approximate form

$$
\begin{gathered}
\widetilde{E}_{\mathrm{d}}(T) \approx \\
\left\{E_{\mathrm{d}}^{2}+\frac{\hbar u^{2} D_{\mathrm{o}}^{2}}{2 k_{\mathrm{B}} T \omega_{\mathrm{o}}}\left[\left(n\left(\omega_{\mathrm{o}}\right)+1\right) \theta\left(1-\frac{\hbar \omega_{\mathrm{o}}}{k_{\mathrm{B}} T}\right) \sqrt{1-\frac{\hbar \omega_{\mathrm{o}}}{k_{\mathrm{B}} T}}+n\left(\omega_{\mathrm{o}}\right) \sqrt{1+\frac{\hbar \omega_{\mathrm{o}}}{k_{\mathrm{B}} T}}\right]\right\}^{1 / 2}
\end{gathered}
$$

explaining the results of the experiment for $\mathrm{MgF}_{2}[4,5]$. Indeed, $\widetilde{E}_{\mathrm{d}}(T)$ is seen to tend to $E_{\mathrm{d}}$, the acoustic deformation-potential constant of Ps, at low temperatures $T \ll \hbar \omega_{\mathrm{o}} / k_{\mathrm{B}}$, where optic vibrations are not excited. In the opposite case, when $T \gg \hbar \omega_{\mathrm{o}} / k_{\mathrm{B}}, \widetilde{E}_{\mathrm{d}}(T)$ tends to the temperature independent effective constant $\widetilde{E}_{\mathrm{d}}=\sqrt{E_{\mathrm{d}}^{2}+\left(u D_{\mathrm{o}} / \omega_{\mathrm{o}}\right)^{2}}$ representing the acoustic deformation-potential constant "renormalized" by nonpolar optic scattering of Ps. One can estimate the ratio $D_{\circ} / \omega_{\circ}$ by comparing this constant with the experimental result for $\mathrm{MgF}_{2}$ at $T>355 \mathrm{~K}(16 \mathrm{eV}$ above $355 \mathrm{~K}, 7.6 \mathrm{eV}$ below $200 \mathrm{~K}$ [4]). One obtains $D_{\circ} / \omega_{\circ} \sim 2 \times 10^{-5} \mathrm{eV} \mathrm{s} / \mathrm{cm}$ then. If one further assumes $\omega_{\mathrm{o}}$ to be $\sim 5 \times 10^{13} \mathrm{~s}^{-1}$, the average acoustic frequency in $\mathrm{MgF}_{2}$ corresponding to the edge of the Brillouin zone (estimated by means of $\omega_{0} \sim u \pi /\left(2 a / 3+c / 3\right.$ ) with $u \approx 7 \times 10^{5} \mathrm{~cm} / \mathrm{s}$ [14] and the $\mathrm{MgF}_{2}$ lattice constants $a=4.64 \AA, c=3.06 \AA[15]$ ), then one obtains the reasonable estimate $D_{\circ} \sim 1 \times 10^{9} \mathrm{eV} / \mathrm{cm}$ for the optic deformation-potential constant of $\mathrm{Ps}$ in $\mathrm{MgF}_{2}$. (Typical values of $D_{\mathrm{o}}$ for electrons and holes in semiconductors are $\sim 5 \times 10^{8} \mathrm{eV} / \mathrm{cm}[7]$; for example, $D_{\circ}=7 \times 10^{8} \mathrm{eV} / \mathrm{cm}$ for electrons in $\mathrm{Ge}[16]$.) 


\section{Conclusions}

Above it was shown that nonpolar scattering of Ps on long-wave optic phonons is responsible for the renormalization of the Ps acoustic deformation-potential constant. This explains drastic temperature broadening of the Ps momentum distribution in $\mathrm{MgF}_{2}$ above $200 \mathrm{~K}$ reported in Refs. [4, 5]. Experimental evidence for the absence of the same effect in $\alpha-\mathrm{SiO}_{2}$ can be attributed to local symmetry restrictions ruling out the nonpolar scattering mechanism in this crystal.

Optic deformation-potential scattering is known to be allowed in degenerate and forbidden in nondegenerate $\Gamma$-valleys (Brillouin zone center) of cubic crystal lattices $[7,13]$. This is easy to understand on the basis of general principles of group theory. The constant $D_{\circ}$ is determined by the matrix element of the perturbation operator, that is the variation of the rigid-lattice potential due to optic lattice vibrations, taken over Bloch wave-functions of a particle in the neighborhood of its band minimum $[12,13]$. For non-degenerate $\Gamma$-valleys of cubic lattices this matrix element transforms according to the unit representation of the point group of the crystal, whereas zone-center optic phonon modes are triply degenerate, i.e. corresponding normal lattice vibrations transform according to the three-dimensional group representation. In such a situation, the interaction (Eq. (6)) is not an invariant with respect to point group transformations and, therefore, vanishes (or, in other words, $D_{\mathrm{o}} \equiv 0$ ). This is the reason why optic deformation-potential scattering is forbidden in nondegenerate and allowed in triply degenerate $\Gamma$-valleys of cubic crystals. If, further, the point group of the crystal has lower symmetry than cubic (noncubic crystals), then the three-dimensional group representation, according to which zone-center optic phonon modes transform, will be reducible. If this reducible representation contains irreducible one of the same dimensionality as the degeneracy multiplicity of the $\Gamma$-valley, then the optic modes transforming according to this irreducible representation will be present in the interaction (Eq. (6)) providing its invariance with respect to point group transformations. The constant $D_{\mathrm{o}}$ is non-zero for such optic modes and these modes will be responsible for nonpolar optic scattering of the particle in the $\Gamma$-valley.

The aforesaid results let one predict a quite new effect for the delocalized $\mathrm{Ps}_{\mathrm{s}}$ atom in crystalline quartz. Crystalline quartz is known to undergo the second-order transition from $\alpha$ - to $\beta$-phase $\left(\alpha-\mathrm{SiO}_{2} \rightarrow \beta-\mathrm{SiO}_{2}\right)$ at temperature above $846 \mathrm{~K}[15,17]$. As this takes place, the type of the symmetry of the quartz lattice increases from $D_{3}$ to $D_{6}[17]$. The $D_{6}$ point symmetry group is isomorphic to the $C_{6 v}$ point group of wurtzite-structure crystals and, therefore, has the same set of group representations for the Brillouin zone center and, consequently, the same selection rules for zone-center optic phonons. According to Ref. [13], the $C_{6 v}$ point group (and $D_{6}$ isomorphic to $C_{6 v}$ ) admits nondegenerate and doubly degenerate $\Gamma$-valleys of the Brillouin zones of corresponding crystals, whereas zone-center optic phonon modes transform according to the group representations of single and double dimensionalities. Therefore, whatever the degeneracy multiplicity of the $\Gamma$-valley, there always exists the optic mode transforming according to the group representation of the dimensionality equal to the degeneracy multiplicity of the $\Gamma$-valley, so that the interaction (Eq. (6)) will always be non-zero for $\Gamma$-valleys 
of crystals of the $C_{6 v}$ and $D_{6}$ point symmetry groups. From this it follows that, being absent in $\alpha-\mathrm{SiO}_{2}$ crystal, Ps nonpolar optic phonon scattering must manifest itself above $846 \mathrm{~K}$, the temperature of $(\alpha-\beta)$-phase transition, yielding the effect of drastic temperature broadening of the $\mathrm{Ps}$ momentum distribution in $\beta-\mathrm{SiO}_{2}$ crystal.

\section{Acknowledgments}

The author would like to thank Prof. I.D. Feranchuk, Prof. L.V. Keldysh, Prof. L.I. Komarov, and Dr. V.N. Kushnir for useful discussions.

\section{References}

[1] J. Kasai, T. Hyodo, K. Fujiwara, J. Phys. Soc. Jpn. 57, 329 (1988) and refs. therein.

[2] I.V. Bondarev, T. Hyodo, Phys. Rev. B 57, 11341 (1998).

[3] I.V. Bondarev, Phys. Rev. B 58, 12011 (1998).

[4] Y. Nagai, M. Kakimoto, H. Ikari, T. Hyodo, Mater. Sci. Forum 255-257, 596 (1997).

[5] Y. Nagai, M. Kakimoto, T. Hyodo, K. Fujiwara, H. Ikari, M. Eldrup, A. Stewart, Phys. Rev. B 62, 5531 (2000).

[6] G.D. Mahan, Many-Particle Physics, Plenum, New York 1981.

[7] B.K. Ridley, Quantum Processes in Semiconductors, Clarendon, Oxford 1982.

[8] O.V. Boev, K.P. Arefiev, Izv. Vyssh. Uchebn. Zaved. Fiz. 25, 118 (1982) [Sov. Phys. J. 25, 37 (1982)].

[9] A.S. Davydov, Theory of Solids, Nauka, Moscow 1976 (in Russian).

[10] I.V. Bondarev, Pis'ma Zh. Eksp. Teor. Fiz. 69, 215 (1999) [JETP Lett. 69, 231 (1999)].

[11] Y. Toyozawa, Prog. Theor. Phys. 26, 29 (1961).

[12] G.L. Bir, G.E. Pikus, Fiz. Tverd. Tela 2, 2287 (1960) [Sov. Phys.-Solid State 2, 2039 (1961)].

[13] G.L. Bir, G.E. Pikus, Symmetry and Deformation Effects in Semiconductors, Nauka, Moscow 1972 (in Russian).

[14] K.S. Aleksandrov, L.A. Shabanova, V.I. Zinenko, Phys. Status. Solidi. 33, K1 (1969).

[15] American Institute of Physics Handbook, Ed. D.E. Gray, McGraw-Hill, New York 1972.

[16] M. Neuberger, Handbook of Electronic Materials, Plenum, New York 1971.

[17] Acoustic Crystals, Ed. M.P. Shaskolskaya, Nauka, Moscow 1982 (in Russian). 